P112 LUPUS NEPHRITIS IN MALES: CLINICAL AND HISTOLOGICAL PROFILE, DISEASE COURSE AND MANAGEMENT - RESULTS FROM A NEPHROLOGY CLINIC IN SRI LANKA

Duminda Basnayake, Abdul Wazil, Nishantha Nanayakkara, Mohommed Shafraz. Nephrology and Transplant Unit, Teaching Hospital Kandy, Kandy, Sri Lanka

10.1136/lupus-2020-eurolupus. 156

Background Lupus Nephritis (LN) in males is poorly understood due to the low prevalence in men. Our aim was to identify the clinical and histological profile on initial presentation and to report the experience of disease course and management.

Methods Seventeen biopsy proven LN males from nephrology clinic, Teaching Hospital Kandy were retrospectively analyzed.

Results The median age at the time of renal biopsy was 25.7 years (range, 14-57 years). Commonest initial presentation was nephrotic syndrome in 10 of 17 patients followed by rapidly progressive glomerulonephritis (RPGN) in 4 and nephritic syndrome in 3 .

Anemia was noted in 11 patients. Thrombocytopenia was seen among 4 patients. ANA report was positive in 13 and negative in 4 patients. Diffuse LN was the commonest histological pattern accounting to 10 cases followed by Focal LN in 4, membranous $\mathrm{LN}$ in 2 and mesangial proliferative $\mathrm{LN}$ in 1 patient.

After remission induction treatment, 10 patients achieved complete renal remission. Five patients achieved partial remission while two patients did not achieve remission. Induction was done with intravenous (IV) methyl prednisolone $(0.5 / 1.0 \mathrm{~g})$ daily 3 doses and IV cyclophosphamide $\left(750 \mathrm{mg} / \mathrm{m}^{2}\right.$ body surface) 2 doses two weeks' apart and 4 doses monthly. Maintenance treatment was with oral prednisolone and mycophenolate mofetil.

Median follow-up of the cohort was 52 months (2-89 months). During the follow-up repeat biopsy was performed in 4 patients. The changes observed were, class shift from class III to IV in 2 patients and from class V to class IV in one patient. Only one patient required renal replacement therapy during the follow up period. Six patients developed chronic kidney disease (CKD) after a median of 33 months follow-up.

Conclusion Commonest presentation of $\mathrm{LN}$ in males is nephrotic syndrome, and type IV LN is the most frequent histological form. Pattern of initial presentation is seemingly associated with the renal outcome. Better delineation needs further studies with larger cohorts.

\section{P113 ONLINE CASE-BASED EDUCATION SUBSTANTIALLY IMPROVED RHEUMATOLOGISTS' CONFIDENCE AND COMPETENCE IN MANAGING SLE AND SELECTING APPROPRIATE TREATMENTS}

${ }^{1}$ Elaine Bell, ${ }^{2}$ Robert McCarthy, ${ }^{3}$ Ronald van Vollenhoven. ${ }^{1}$ Articulate Medical Communications Ltd, Hitchin, UK; ${ }^{2}$ Medscape LLC, New York, USA; ${ }^{3}$ Amsterdam Rheumatology and Immunology Center ARC, Amsterdam, The Netherlands

10.1136/lupus-2020-eurolupus. 157

Background/Purpose The prognosis for patients with systemic lupus erythematosus (SLE) has improved in recent years, but many patients fail to achieve optimal disease control. We assessed whether an online case-based CME activity could improve rheumatologists' confidence in managing SLE and in selecting appropriate treatments for their patients.

Methods Rheumatologists participated in a segmented online multi-media activity consisting of videos portraying realistic physician-patient interaction followed by a test question and expert commentary. Educational effect was assessed using a repeated-pair design, pre-/post-assessment. A Chi-square test of independence determined if a statistically significant improvement $(5 \%$ significance level, $P<0.05)$ existed in the number of correct responses between the pretest and posttest scores. Cramer's V estimated the effect size of the education. The activity launched 26 March 2019 with data collection through 29 April 2019.

Results

- $35 \%$ of rheumatologists' $(n=46)$ answered all 3 questions correctly at baseline rising to $85 \%$ post assessment $(\mathrm{P}<.001)$

- Significant improvement in knowledge of the proportion of SLE patients testing positive for anti-nuclear antibodies $(72 \%$ at baseline, 93\% post assessment; $\mathrm{P}<.01$ ), and significant improvement in treatment choice for a patient who experienced worsening symptoms following initial success with prednisone, hydroxychloroquine and methotrexate $(59 \%$ at baseline, 91\% post asessment; $\mathrm{P}<.001$ )

- Extensive educational impact (Cramer's V $=0.277$ ) on knowledge and competence overall with $30 \%$ of rheumatologists reporting greater confidence in their ability to treat SLE symptoms

- $38 \%$ of participants reported an intent to make changes in their practice based on what they learned; of those who intended to make a change, $92 \%$ were highly committed to implementing these changes

Conclusions Participation in this segmented online multi-media activity was highly effective in improving rheumatologists' confidence and competence in treatment selection and management of SLE. The extensive impact of this education should translate into improvements in clinical practice.

Acknowledgement Supported by GlaxoSmithKline

\section{P114 EVALUATION OF NEUROPSYCHIATRIC INVOLVEMENT AND PSYCHOMETRIC STATUS IN PEDIATRIC SYSTEMIC LUPUS ERYTHEMATOSUS (SLE)}

Emil Aliyev, Yelda Bilginer. Pediatric Rheumatology Dept., Hacettepe University Faculty of Medicine, Turkey

\subsection{6/lupus-2020-eurolupus. 158}

Background Systemic Lupus Erythematosus (SLE) is an autoimmune disease characterized by multiple organ involvement. Neuropsychiatric involvement is seen in $20-27 \%$ of patients. Approximately $1 / 4$ of diagnosed SLE patients develop neuropsychiatric SLE 2 years after diagnosis.

Methods The aim of this study was to determine the risk groups before developing neurological, psychiatric and cognitive involvement and symptoms in SLE patients and to take measures and to develop cheaper and more applicable algorithms.

Results Ninety eight children and adolescents were included in the study. $61(62.2 \%)$ of the patients were female and 37 (37.8\%) were male. Research group included 34 (34.7\%) SLE patients, 31 (31.6\%) JIA patients, and 33 (33.7\%) healthy children and adolescents. The median age of the patients was 17 (12-21) years, and the median follow-up period was 4 
years (0-13). The median SLEDAI of the patients was 2 points (0-19). As a result of psychiatric screening, separation anxiety disorder, generalized anxiety disorder and specific phobia were significantly more common in SLE patients than in healthy controls (respectively $p=0,054, p=0,052, p=0,018$ ). The Wechsler Intelligence Scale for Children-IV (WISC-IV) was applied to children and adolescents. In the test results, Perceptual Reasoning Converted Index (PRCI) and Perceptual Reasoning Standard Index (PRSI) were significantly lower in SLE patients compared to the healthy control group (respectively $\mathrm{p}=0,039, \mathrm{p}=0,046$ ). The 'comprehension' subtest, 'symbol search' subtest and Verbal Comprehension Standard Index (VCSI) were found significantly lower in SLE patients compared to the healthy control group (respectively $\mathrm{p}=0,046$, $\mathrm{p}=0,017, \mathrm{p}=0,036)$.

Conclusion This study is the first in the literature about this field. It is important to show early neuropsychiatric involvement with neurocognitive and neuropsychological tests in pediatric SLE patients. We also examined the incidence of psychiatric diagnosis in SLE patients. SLE patients should be evaluated with neurocognitive and neuropsychological tests regardless of disease activity, even though they do not have neurological signs and symptoms. Patients should be closely monitored for neuropsychiatric involvement and tests should be repeated if necessary.

Keywords Systemic Lupus Erythematosus (SLE), SLEDAI, Neuropsychiatric SLE (NPSLE), neurocognitive disorder, neuropsychological assessment, psychometric test, psychiatric screening, WISC-IV, WISC-IV subtests, closters of WISC-IV score

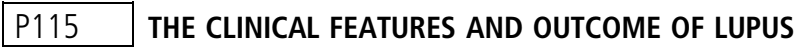 NEPHRITIS PATIENTS PRESENTING WITH MYOCARDITIS: A SINGLE CENTER EXPERIENCE}

\begin{abstract}
${ }^{1,2}$ Nouran Eshak, ${ }^{1}$ Shaimaa Shoela, ${ }^{1}$ Fatma Fayed, ${ }^{1}$ Asmaa Beltagy, ${ }^{1}$ Mai Morsy, ${ }^{3}$ Mahmoud Abdelnabi. ${ }^{1}$ Rheumatology Unit, Dept. of Internal Medicine, Faculty of Medicine, University of Alexandria, Egypt; ${ }^{2}$ Internal medicine Dept., Texas Tech University Health Science Center, Texas, USA; ${ }^{3}$ Cardiology and Angiology Unit, Dept. of Clinical and Experimental Internal Medicine, Medical Research Institute, University of Alexandria, Egypt
\end{abstract}

\subsection{6/lupus-2020-eurolupus. 159}

Background/Objectives Lupus Myocarditis (LM) is one of the rare, but life-threatening manifestations of SLE. The objective was to describe the clinical, laboratory and echocardiographic findings, management, and outcome of 10 Lupus Nephritis (LN) patients presented with LM.

Patients and Methods A retrospective analysis of data of patients presented to our tertiary care center with LN and new onset myocardial involvement from June 2017 till May 2019, was done. All patients fulfilled the SLICC criteria for the diagnosis of SLE. LM was diagnosed by a combination of new-onset cardiac symptoms and echocardiography showing myocardial involvement in the form of global/segmental hypokinesia and reduced left ventricular ejection fraction (LVEF).

Results Regarding baseline characteristics, there were 10 patients with 8 females, with a mean age of 22.3 (15-31) years and mean duration of SLE diagnosis of $3.8(0-12)$ years. 2 patients had a history of hypertension, while none of them had a previous cardiac history or DM.

Regarding the onset of LM, in 6 patients it coincided with a diagnosis of $\mathrm{LN}$, in the others, LN preceded LM with a 2year mean interval.

\section{Abstract P115 Table 1}

\begin{tabular}{|c|c|c|c|}
\hline \multicolumn{2}{|c|}{ 1- Baseline patient characteristics $(n=10)$} & & \\
\hline Age (years) & 22.3 years & & \\
\hline Female Sex & $8(80 \%)$ & & \\
\hline SLE diagnosis duration & 3.8 years & & \\
\hline History of hypertension & $2(20 \%)$ & & \\
\hline History of DM & $0(0 \%)$ & & \\
\hline Previous cardiac history & $0(0 \%)$ & & \\
\hline \multicolumn{4}{|l|}{ 2- Clinical data } \\
\hline \multirow[t]{2}{*}{ Onset of LM } & LN and LM & & $\mathrm{LN}$ preceding $\mathrm{LM}$ \\
\hline & $6(60 \%)$ & & $4(40 \%)$ \\
\hline \multirow[t]{2}{*}{ Clinical presentation } & Cardiogenic shock & Acute heart failure & Syncope \\
\hline & $4(40 \%)$ & $5(50 \%)$ & $1(10 \%)$ \\
\hline
\end{tabular}

\begin{tabular}{lll}
\multicolumn{2}{c}{ 3- Laboratory and renal biopsy data } & \\
CBC & HB level & $8.7 \mathrm{gm} / \mathrm{dl}$ \\
& Wbcs count & $6.110^{\wedge} 3 / \mu \mathrm{l}$ \\
& Platelets count & $19910^{\wedge} 3 / \mu \mathrm{l}$ \\
Creatinine level & $2.6 \mathrm{mg} / \mathrm{dl}$ & \\
Protein: Creatinine ratio & $3.6 \mathrm{mg} / \mathrm{mg}$ & \\
Patients with renal biopsy & $5(50 \%)$ & \\
& Class III & Class IV \\
& $1(20 \%)$ & $4(80 \%)$
\end{tabular}

4- Baseline Echocardiography

\begin{tabular}{ll} 
LVEF & $26.3 \%$ \\
ESD & $46.1 \mathrm{~mm}$ \\
EDD & $56.3 \mathrm{~mm}$ \\
Global hypokinesia & $9(90 \%)$ \\
Mitral regurgitation & $10(100 \%)$ \\
Tricuspid regurgitation & $8(80 \%)$ \\
Pericardial effusion & $7(70 \%)$ \\
Pulmonary hypertension & $4(40 \%)$ \\
\multicolumn{1}{|c|}{ 5- Management } & $10(100 \%)$ \\
Pulse steroids & $7(70 \%)$ \\
IV cyclophosphamide & $3(30 \%)$ \\
mycophenolate mofetil & $1(10 \%)$ \\
Immunoadsorption & \\
\multicolumn{1}{r}{ 6- Follow-up echocardiography after 3 months } \\
LVEF & $42.8 \%$ \\
ESD & $38.9 \mathrm{~mm}$ \\
EDD & $52 \mathrm{~mm}$ \\
7- Outcomes & \\
Partial improvement & $2(20 \%)$ \\
Mortality & $3(30 \%)$ \\
\hline
\end{tabular}

Data is represented as mean or number (\%)

Regarding clinical presentation, 4 patients presented with cardiogenic shock, while 5 had symptoms of acute heart failure and 1 had repeated syncope.

Regarding laboratory and renal biopsy data, mean creatinine was $2.6(0.3-9.6) \mathrm{mg} / \mathrm{dl}$, Protein: Creatinine ratio was 3.6 (114) $\mathrm{mg} / \mathrm{mg}$. 5 patients had a renal biopsy, 1 had class III LN, 4 had class IV LN, 2 of them progressed to end-stage renal disease (ESRD) and were on maintenance hemodialysis (MHD) at time of presentation with myocarditis.

Baseline Echocardiography showed mean LVEF of 26.3 $(15-40) \%$, LV end-diastolic dimension (LVEDD) of 56.3 (46- 